\title{
Study of Bone Quality and Fracture Line Morphology of the Posterior Medial Humeral Calcar in Proximal Humeral Fractures
}

\section{Zheng Xu}

Chengdu University of Traditional Chinese Medicine https://orcid.org/0000-0002-0872-6733

\section{Ming Xiang}

Sichuan Provincial Orthpaedics Hospital

Jinsong Yang

Sichuan Provincial Orthpaedics Hospital

\section{Xu Gao}

Chengdu University of Traditional Chinese Medicine

Yi Cao ( $\nabla 29235675 @ q q . c o m$ )

Chengdu University of Traditional Chinese Medicine Affiliated Hospital https://orcid.org/0000-00034252-6476

\section{Research article}

Keywords: bone quality, fracture line morphology, posterior medial humeral calcar, proximal humeral fractures, investigate, patients, statistical analysis

Posted Date: October 5th, 2021

DOl: https://doi.org/10.21203/rs.3.rs-934274/v1

License: @ (i) This work is licensed under a Creative Commons Attribution 4.0 International License. Read Full License 


\section{Abstract}

Objective: To investigate the relationship between fracture line morphology, bone quality and fracture morphology of the posterior medial humeral calcar in proximal humeral fractures

METHODS: CT data of patients with proximal humeral fractures diagnosed in our hospital from 06/2019 to $06 / 2021$ were retrospectively analyzed to describe the map and coordinate analysis of the posterior medial humeral calcar fracture based on three-dimensional reconstruction, to create varus, valgus and normal groups according to the inclination angle of the humeral head, and to measure the bone mass of the posterior medial humeral calcar and perform statistical analysis.

RESULTS: Sixty-two patients met the inclusion criteria, aged 15 to 72 years, there were 21 varus, 24 valgus and 17 normal types. Epiphyseal extension occurred most frequently posteriorly and medially, with the fracture line ending mostly posterior to the greater tuberosity. The thickness of the varus was (2.33 \pm $0.47)$, valgus was $(2.59 \pm 0.33)$ and normal type was $(2.69 \pm 0.53)$. The T-test showed that the thickness of the bone in the varus was less than the other two types, and bone density and pinch angle were no statistically significant.

CONCLUSION: There is no correlation between the trend of the posterior medial fracture line of the humeral calcar, bone density and internal, external rotation of the humeral head. The medial bone thickness after varus is less than that of valgus and normal fractures. The fracture line endpoint is mostly located posterior to the greater tuberosity.

\section{Introduction}

Proximal humeral fractures are the third most common fracture in the elderly, and their incidence increases each year as society ages, with the incidence expected to triple in the next 30 years[1]In recent years, with the introduction of the calcar screw[2] and Russo's "four-plane fractionation"[3], which refers to the humeral calcar as the "disappearing fifth fragment, " the humeral calcar's clinical significance in the treatment of proximal humeral fractures continues to grow. The humeral calcar is a segment of the bone cortex below the humerus head and is often involved in fractures of the second, third and fourth parts of the proximal humerus, often with comminution.

The proximal humerus has a cervical stem angle of approximately $130^{\circ}$ and a $30^{\circ}$ posterior inclination. When a "parachute reflex" [4] fracture of the proximal humerus occurs, it often involves the posterior medial aspect of the humeral calcar. The majority of proximal humeral operations are currently performed using a deltoid - pectoralis central approach. In this case, the medial-posterior humeral calcar is relatively under-exposed and is challenging to fix internally, resulting in a suboptimal repositioning of the posterior medial humeral calcar.

A better understanding of where fracture lines commonly occur may influence all aspects of surgical treatment, including exposure, repositioning techniques and internal fixation design. Some anatomical 
studies[5] using arterial injections have found that perfusion of the head can be accomplished through a small branch of the posterior rotator humeral artery within the posterior medial periosteum.

Campochiaro[6] states that soft tissue stripping, especially posterior medial, must be avoided as much as possible during repositioning internal fixation to avoid ischaemic necrosis, so we need to clarify the alignment of the fracture line on the posterior medial humeral calcar to avoid affecting the soft tissues during repositioning of the fracture block Hetrel[7] notes that the predictors of humeral head ischaemia: the length of the epiphyseal extension, the medial hinge, is most often located posteriorly medially, reflecting some extent the association between the course of the posterior medial humeral fracture line and the blood supply to the humeral head. In terms of bone quality, morphometrics has noted that bone strength is primarily related to the number of trabecular connections for the proximal humerus. The most significant bone mass and bone volume are observed medially and posteriorly to the bone and are crucial for obtaining supported repositioning[8].

Therefore, we focused on the posterior medial aspect of the humeral calcar. However, We looked through the literature that there is no literature on the posterior medial aspect of the humeral calcar.

The CT imaging data of patients with proximal humeral fractures admitted from Jun 2019 to Jun 2021 are reviewed and analysed to study the relationship between the posterior medial fracture line morphology, cortical bone quality and fracture morphology of proximal humeral fractures through CT 3D reconstruction to deepen clinicians' further understanding and knowledge of the posterior medial humeral calcar and to improve the proximal humeral fractures to a certain extent. The aim is to improve the treatment outcome of proximal humeral fractures.

\section{Materials And Methods}

\section{Subjects}

The inclusion criteria were as follows: 『lmaging confirmed proximal humeral fracture involving the humeral calcar $₫$ Complete and clear preoperative CT data $₫$ Low energy injury $\otimes$ Normal humeral development

The exclusion criteria were as follows: $₫$ pathological fracture $\otimes$ previous shoulder fracture $\otimes$ severe comminution of the humeral calcar $\otimes$ severe osteoporosis

\section{CT data acquisition}

Ninety-nine patients who had a CT scan and were diagnosed with a proximal humeral fracture involving the humeral calcar were selected from 06/2020 to 06/2021. All patients were scanned with 64-row spiral $\mathrm{CT}$. The scans covered the area from the clavicle to the lower humeral segment. The case data was saved in Dicom format via the imaging system.

\section{Fracture block modelling, virtual repositioning}


The DICOM format file was imported into Mimics 20.0, the cortical bone threshold was set, and the resulting 3D image was exported in STL format using the region growing, wrap and smooth functions (Figs. 1 and 2).

Import the STL file into Rhino 6, select the right humeral view for all humerus; mirror inverts the left humerus to change to the right, perform a series of segmentation moves etc. to restore the fracture model to an average proximal humeral model, position the humeral volume centre of gravity to the origin, use the most convex point of the greater tuberosity and the most convex point of the deltoid ramus as the bony landmark, rotate the object around the $\mathrm{X}, \mathrm{Y}$ and $\mathrm{Z}$ axes so that the most convex point of the more excellent tuberosity Position the object in the lower part of the XZ plane, with the $X$-axis crossing the midline of the volume. Position the most convex point of the trochanteric tuberosity in the $\mathrm{YZ}$ plane to ensure the uniformity of the world coordinates of the supraspinatus point (Fig. 3). Combine the parietal and fluoroscopic windows and intercept the bitmap image on the posterior medial aspect of the humeral calcar.

\section{Morphological analysis}

\subsection{Fracture mapping}

The humeral calcar and fracture line were drawn at the repositioned humeral calcar, and a standard proximal humeral CT was selected to model and simulate the repositioning as a template as described above. All patient intercepts were superimposed on the humeral calcar template using Fireworks CS6 to obtain a fracture line distribution (Fig. 4).

We positioned the humeral calcar fracture line start point anterior to the humerus and the fracture line endpoint posterior to the humerus and made and recorded world coordinates at the fracture line start point, endpoint and corner points. The coordinates of the points were imported into a written Matlab R2015 script, and 2D and 3D heat maps were drawn to determine the range of the humeral calcar fracture line start and endpoints (Figs. 13 and 14), and the range was placed into the proximal humeral template to describe the fracture-prone zone of the humeral calcar (Figs. 15 and 16).

\subsection{The angle of inclination of the humeral head}

So far most people measure the inclination angle of the humeral head concerning Hetrel's[9] method and based on orthogonal x-rays of the shoulder joint, which is clinically convenient for observation and diagnosis, but the humerus as a three-dimensional object is inaccurate when measured only by a twodimensional image such as an x-ray. Therefore, I propose a new three-dimensional measurement scheme (Fig. 5): import the STL file exported from mimics into 3-Matic 12.0, mark the articular surface of the humeral head, fit a circular body using sphere fitting, make points at three equal points on the edge of the articular surface to fit a closed curve, connect the centre of gravity of the sphere to the centre of the curve (line A), and fit the humeral stem to a cylinder using cylinder fitting. A cylinder with the volume midline of the cylinder (straight line B) measuring the spatial angle with straight-line A. Before using the above method, the angle of inclination of the $x$-slice was measured separately from the above spatial angle 
using multiple cases of the normal proximal humerus, and the error was demonstrated to be within $2^{\circ}$, provided that human error was excluded.

\subsection{The angle between fracture line and horizontal line}

Using the intercepted bitmap image (Fig. 6), the highest point of the fracture line above the humerus is made all straight lines, and similarly, the lowest point of the fracture line below the humerus is made all straight lines. The two straight lines are taken to be the median line and the intersection of the median line with the most posterior aspect of the fracture line and the starting point of the fracture line (straight line A) to show the average trend of fracture line travel. A vertical line (line B) is made across the starting point of the fracture line to show the horizontal plane of the image. The angle between line $A$ and line $B$ is defined as the angle between the fracture line and the horizontal plane of the humerus.

\section{Mechanics and quality analysis 5.1 Finite element analysis}

The proximal humeral template was imported into Geomagic 2013 reverse engineering software for smoothing and filling (Fig. 7), and the data was imported into Ansys 2019 for static analysis (Figs. 8 and 9 ). Based on the data from previous studies $[10,11]$, the modulus of elasticity of cortical bone was 16,000 $\mathrm{MPa}$, and the Poisson's ratio was 0.3. The distal humeral fixation was set (Fig. 10), and the combined forces on the glenohumeral joint surface were most significant at 90 degrees of shoulder abduction (Fig. 11), by Poppen's method[12] better reflects the stresses on the humeral calcar. A pressure of $600 \mathrm{~N}$ was applied to the glenohumeral joint surface to observe the humeral calcar stresses and deformation.

\subsection{Bone cortical bone density and thickness analysis}

After 3D modelling in mimics as described above, the 3D navigational locator was positioned with the proximal humerus and measured on the sagittal CT image using the circular zone delineation tool to measure the mean $\mathrm{HU}$ value and calculate the manifest density concerning Rho's[13] formula for the relationship between manifest density and Hu value. $\rho \rho(\mathrm{g} \cdot \mathrm{cc} \mathrm{mm}-3)=0.131+(0.000624 * \mathrm{HU})$, the manifest density was calculated. The wall thickness was analysed using the wall thickness analysis function of 3-Matic 12.0 (Fig. 12), dividing the wall thickness into six intervals and taking the average of the lowest and highest values of the interval it was located.

\section{Statistical analysis}

SPSS 26.0 statistical software was used for statistical processing. Internal and external overturning was used as the dependent variable, and bone cortical expression density, bone thickness and angle to the fracture level were used as independent variables for two-by-two comparison and independent samples Ttest analysis, where internal overturning was defined as head tilt angle $<130^{\circ}$, external overturning as head tilt angle $>140^{\circ}$ and normal as $>130^{\circ}<140^{\circ}$ according to Mazzucchelli[14]. 


\section{Results}

\section{General results}

The group consisted of 27 males and 35 females; aged 15 to 72 years. There were 30 cases on the left side and 32 cases on the right side. All were the result of low-energy injuries from falls in life. There were 21 cases of varus, 24 cases of valgus and 17 cases of the regular type.

\section{Features of the posterior medial humeral calcar fracture 2.1 Fracture line alignment}

There were significantly more intracapsular than extracapsular fractures of the humeral calcar, consistent with Hasan's[15] study. Epiphyseal extension occurs most often posteriorly and medially in line with Hertel's[7] findings (Fig. 4). It has also been noted in the literature[16] that most cortical thinning occurs posterior to the articular margin so that as stress increases, the fracture line travels along the thinthickness divide because of the varying degrees of thinness and the change in stress in our study (Fig. 9) could also explain this phenomenon.

The coordinates of the fracture line start and endpoints were found in conjunction with the humeral template to confirm the position: fracture line start $x$-axis range $(-25-11), y$-axis range $(-20-15), z$-axis range (-5-15); fracture line end $x$-axis range $(-24-10)$, $y$-axis range (5-17), $z$-axis range $(-5-20)$. The origin is primarily located in the anterior aspect of the humeral joint, and the fracture line endpoint is located chiefly posterior to the greater tuberosity (Figs. 15 and 16).

\subsection{Bone cortical thickness, density and relationship to varus and valgus of the fractured head}

Wall thickness analysis revealed that the medial and dorsal humeral calcar thickness was generally higher than the other regions, consistent with the Hepp[8] results. Based on the statistical results, we found that the posterior medial cortical thickness of the humeral calcar was statistically significant $(\mathrm{p}<$ 0.05 ) with varus and that the cortical thickness of varus was less than that of both valgus and normal fractures (Table 1). 
Table 1

T-test of the posterior medial humeral calcar (bone thickness, density, pinch angle) with varus and valgus.

\begin{tabular}{|lllllll|}
\hline $\begin{array}{l}\text { Measured } \\
\text { values }\end{array}$ & Group & $\begin{array}{l}\text { Varus } \\
(\mathbf{n = 2 1 )}\end{array}$ & $\begin{array}{l}\text { Valgus } \\
(\mathbf{n = 2 4 )}\end{array}$ & $\begin{array}{l}\text { Normal } \\
(\mathbf{n}=\mathbf{1 7})\end{array}$ & $\mathbf{P}$ & \\
\hline & 1 & $0.58 \pm 0.11$ & $0.56 \pm 1.14$ & & 0.422 & 0.675 \\
\hline Density & 2 & $0.58 \pm 0.11$ & & $0.61 \pm 0.11$ & -0.797 & 0.431 \\
\hline & 3 & & $0.56 \pm 1.14$ & $0.61 \pm 0.11$ & -1.098 & 0.279 \\
\hline & 1 & $2.33 \pm 0.47$ & $2.59 \pm 0.33$ & & -2.085 & 0.043 \\
\hline Thickness & 2 & $2.33 \pm 0.47$ & & $2.69 \pm 0.53$ & -2.134 & 0.040 \\
\hline & 3 & & $2.59 \pm 0.33$ & $2.69 \pm 0.53$ & -0.660 & 0.516 \\
\hline Clamp angle & 2 & $4.53 \pm 14.13$ & & $-5.63 \pm 20.02$ & 1.834 & 0.075 \\
\hline & 3 & & $-1.87 \pm 9.97$ & $-5.63 \pm 20.02$ & 0.716 & 0.482 \\
\hline
\end{tabular}

\section{Discussion}

To our knowledge, this is the first study to use a three-dimensional reconstruction to describe a fracture map of the posterior medial humeral calcar. Although the fracture map of the proximal humeral fracture Hasan[15] has been studied, its study of the humeral calcar, especially the posterior medial region, is superficial and only indicates that the proximal humeral fracture is extracapsular in the intracapsular region of the humeral calcar, which is consistent with our findings. At the same time, we hope to explain part of the fracture mechanism using the finite element method in conjunction with bone mass, which has been increasingly used in the field of orthopaedic biomechanics.

The integrity of the humeral calcar is of great importance to the blood supply to the humeral head[17]. The blood supply to the humeral head from the posterior rotator humeral artery accounts for $64 \%$. The Hettrich[18] findings found that the posterior rotator humeral artery is not adherent to the humerus and that the posterior aspect of the greater tuberosity is mostly a concentration of the nourishing hole of the posterior rotator humeral artery, in combination with our finding that when the surgical neck is fractured, the posterior medial fracture line will most likely pass through the trophoblastic foramen of the posterior of the greater tuberosity of the humerus. At the same time, the posterior medial fracture line is angled chiefly and has a specific penetrating force, and when the head-stem is displaced, it is straightforward to damage the periosteum.,the posterior rotator humeral artery has small branches within the posterior medial periosteum to head for perfusion. It has been documented that when the head-stem of the humerus is displaced by more than $3 \mathrm{~mm}$, the periosteum of the medial humeral cortex begins to tear. We conjecture that when the posterior medial humeral calcar is fractured, the rear rotor humeral artery is 
affected because the fracture line damages the nourishing hole posterior to the greater tuberosity and the intraperitoneal vessels, indirectly affecting the perfusion of the rear rotor humeral artery to the head, which is consistent with Meyer's[19] theory.

The advantages of locking plates over non-locking plates in the plating treatment of proximal humeral fractures have been clinically and biomechanically proven. However, a Meta-analysis[20] noted that the main postoperative complications of locking plates include internal displacement of the humeral head, screw removal and humeral head necrosis, with the first two accounting for up to $55 \%$ of all complications. When the humeral head is displaced as a varus deformity, more complications occur, clinical outcomes are week, and postoperative surgical failure rates increase significantly[21, 22].

Therefore, how to effectively avoid internal displacement of the humeral head has become an urgent problem. With the introduction of the humeral talar screw, the deficiency of the plate being on the tension side and not being able to support the medial side has been remedied. But can the humeral talar screw do a perfect job of supporting the stress side? Bai[23] points out that there is no biomechanical advantage to using the talar screw for internal humeral deformity, and Osterhoff[24] proves this point. There are currently two ways to facilitate the maintenance of humeral head repositioning, besides the talar screw there is also cortical support repositioning and medial wall support repositioning is better than the use of the talar screw-in maintaining the stability of the humeral head[25].

We found that the thickness of the posterior medial cortex of the varus is less than that of the valgus and ordinary fractures, so attention should be paid to the repositioning of the posterior medial humeral calcar during intraoperative repositioning. At the same time, bone grafting and bone cement can be used to increase the thickness of the posterior medial humeral calcar cortex, restore the continuity of the medial cortex, provide cortical support for the humeral head, avoid the varus of the humeral head after surgery and reduce the occurrence of postoperative complications.

It has been suggested[6] that the humeral calcar should be evaluated in multiple planes of imaging. The operator should have a complete understanding of the morphology of the humeral calcar fracture based on preoperative X-ray and CT findings to determine the surgical plan. This is why we chose to conduct the above study based on 3D reconstruction, which has better visualisation and accuracy in orthopaedic studies. A three-dimensional staging scheme[26] for proximal humeral fractures has been proposed but has not received sufficient attention. Most of the standard research protocols are currently limited to the two-dimensional level due to previous technology, and orthopaedic research in the three-dimensional level needs to be further developed.

\section{Shortcomings and limitations}

All studies in this experiment are based on data in the original DICOM format, the international standard for medical images and related information and are the core of the medical image format. The model constructed conforms to the natural human proximal humeral bone to the maximum possible through professional engineering software. However, due to the inability to simulate the complex stresses on the 
shoulder joint and the exclusion of soft tissues such as muscles and ligaments, there are still some errors with the actual situation.

\section{Conclusion}

After surgical neck fractures of the humerus, there is no correlation between the trend of the posterior medial fracture line of the humeral calcar, bone density and internal, external rotation of the humeral head. The medial bone thickness after varus is less than that of valgus and normal fractures. The fracture line endpoint is mostly located posterior to the greater tuberosity, which may be a factor influencing the blood supply to the humeral head and requires further analysis.

\section{Abbreviations}

Not applicable

\section{Declarations}

Ethics approval and consent to participate: Not applicable.

Consent for publication: Not applicable.

Availability of data and materials: The datasets used and/or analysed during the current study are available from the corresponding author on reasonable request.

Competing interests: The authors declare that they have no competing interests.

Funding:No funding.

Authors' contributions:; JsY analysed the data.XG collected the data.YC and MX critically reviewed the content of the article.ZX implemented the research and drafted the article,and was a major contributor in writing the manuscript. All authors read and approved the final manuscript.

Acknowledgements: Not applicable.

\section{References}

1. Palvanen $\mathrm{M}$, Kannus $\mathrm{P}$, Niemi S, Parkkari J. Update in the epidemiology of proximal humeral fractures. Clin Orthop Relat Res. 2006;442:87-92.

2. Gardner MJ, Weil Y, Barker JU, Kelly BT, Helfet DL, Lorich DG. The importance of medial support in locked plating of proximal humerus fractures. J Orthop Trauma. 2007;21:185-91.

3. Russo R, Cautiero F, Della Rotonda G. The classification of complex 4-part humeral fractures revisited: the missing fifth fragment and indications for surgery. Musculoskelet Surg. 2012;96(Suppl 1):13-9. 
4. Majed A, Thangarajah T, Southgate DF, Reilly P, Bull A, Emery R. The biomechanics of proximal humeral fractures: Injury mechanism and cortical morphology. Shoulder Elbow. 2019;11:247-55.

5. Brooks $\mathrm{CH}$, Revell WJ, Heatley FW. Vascularity of the humeral head after proximal humeral fractures. An anatomical cadaver study. J Bone Joint Surg Br. 1993;75:132-6.

6. Campochiaro G, Rebuzzi M, Baudi P, Catani F. Complex proximal humerus fractures: Hertel's criteria reliability to predict head necrosis. Musculoskelet Surg. 2015;99(Suppl 1):9-15.

7. Hertel R. Fractures of the proximal humerus in osteoporotic bone. Osteoporos Int. 2005;16(Suppl 2):65-72.

8. Hepp P, Lill H, Bail H, Korner J, Niederhagen M, Haas NP, Josten C, Duda GN. Where should implants be anchored in the humeral head? Clin Orthop Relat Res. 2003;415:139-47.

9. Hertel R, Knothe U, Ballmer FT. Geometry of the proximal humerus and implications for prosthetic design. J Shoulder Elbow Surg. 2002;11:331-8.

10. Clavert P, Zerah M, Krier J, Mille P, Kempf JF, Kahn JL. Finite element analysis of the strain distribution in the humeral head tubercles during abduction: comparison of young and osteoporotic bone. Surg Radiol Anat. 2006;28:581-7.

11. Friedman RJ, Laberge M, Dooley RL, O'Hara AL. Finite element modeling of the glenoid component: Effect of design parameters on stress distribution. J Shoulder Elbow Surg. 1992;1:261-70.

12. Poppen Nk Fau. - Walker PS, Walker PS: Forces at the glenohumeral joint in abduction.

13. Rho JY, Hobatho MC, Ashman RB. Relations of mechanical properties to density and CT numbers in human bone. Med Eng Phys. 1995;17:347-55.

14. Mazzucchelli RA, Jenny K, Zdravkovic V, Erhardt JB, Jost B, Spross C. The influence of local bone quality on fracture pattern in proximal humerus fractures. Injury. 2018;49:359-63.

15. Hasan AP, Phadnis J, Jaarsma RL, Bain GI. Fracture line morphology of complex proximal humeral fractures. J Shoulder Elbow Surg. 2017;26:e300-8.

16. Majed A, Thangarajah T, Southgate D, Reilly P, Bull A, Emery R. Cortical thickness analysis of the proximal humerus. Shoulder Elbow. 2019;11:87-93.

17. Kralinger F, Unger S, Wambacher M, Smekal V, Schmoelz W. The medial periosteal hinge, a key structure in fractures of the proximal humerus: a biomechanical cadaver study of its mechanical properties. J Bone Joint Surg Br. 2009;91:973-6.

18. Hettrich CM, Boraiah S, Dyke JP, Neviaser A, Helfet DL, Lorich DG. Quantitative assessment of the vascularity of the proximal part of the humerus. J Bone Joint Surg Am. 2010;92:943-8.

19. Meyer C, Alt V, Hassanin H, Heiss C, Stahl JP, Giebel G, Koebke J, Schnettler R. The arteries of the humeral head and their relevance in fracture treatment. Surg Radiol Anat. 2005;27:232-7.

20. Sproul RC, lyengar JJ, Devcic Z, Feeley BT. A systematic review of locking plate fixation of proximal humerus fractures. Injury. 2011;42:408-13.

21. Hardeman F, Bollars P, Donnelly M, Bellemans J, Nijs S. Predictive factors for functional outcome and failure in angular stable osteosynthesis of the proximal humerus. Injury. 2012;43:153-8. 
22. Oppeboen S, Wikeroy AKB, Fuglesang HFS, Dolatowski FC, Randsborg PH. Calcar screws and adequate reduction reduced the risk of fixation failure in proximal humeral fractures treated with a locking plate: 190 patients followed for a mean of 3 years. J Orthop Surg Res. 2018;13:197.

23. Bai L, Fu ZG, An S, Zhang PX, Zhang DY, Jiang BG. Effect of Calcar Screw Use in Surgical Neck Fractures of the Proximal Humerus With Unstable Medial Support: A Biomechanical Study. J Orthop Trauma. 2014;28:452-7.

24. Osterhoff G, Ossendorf C, Wanner GA, Simmen HP, Werner CM. The calcar screw in angular stable plate fixation of proximal humeral fractures-a case study. J Orthop Surg Res. 2011;6:50.

25. Zhang X, Huang J, Zhao L, Luo Y, Mao H, Huang Y, Chen W, Chen Q, Cheng B. Inferomedial cortical bone contact and fixation with calcar screws on the dynamic and static mechanical stability of proximal humerus fractures. J Orthop Surg Res. 2019;14:1.

26. Edelson G, Kelly I, Fau - Vigder F, Vigder F, Fau - Reis ND, Reis ND. A threedimensional classification for fractures of the proximal humerus.

\section{Figures}




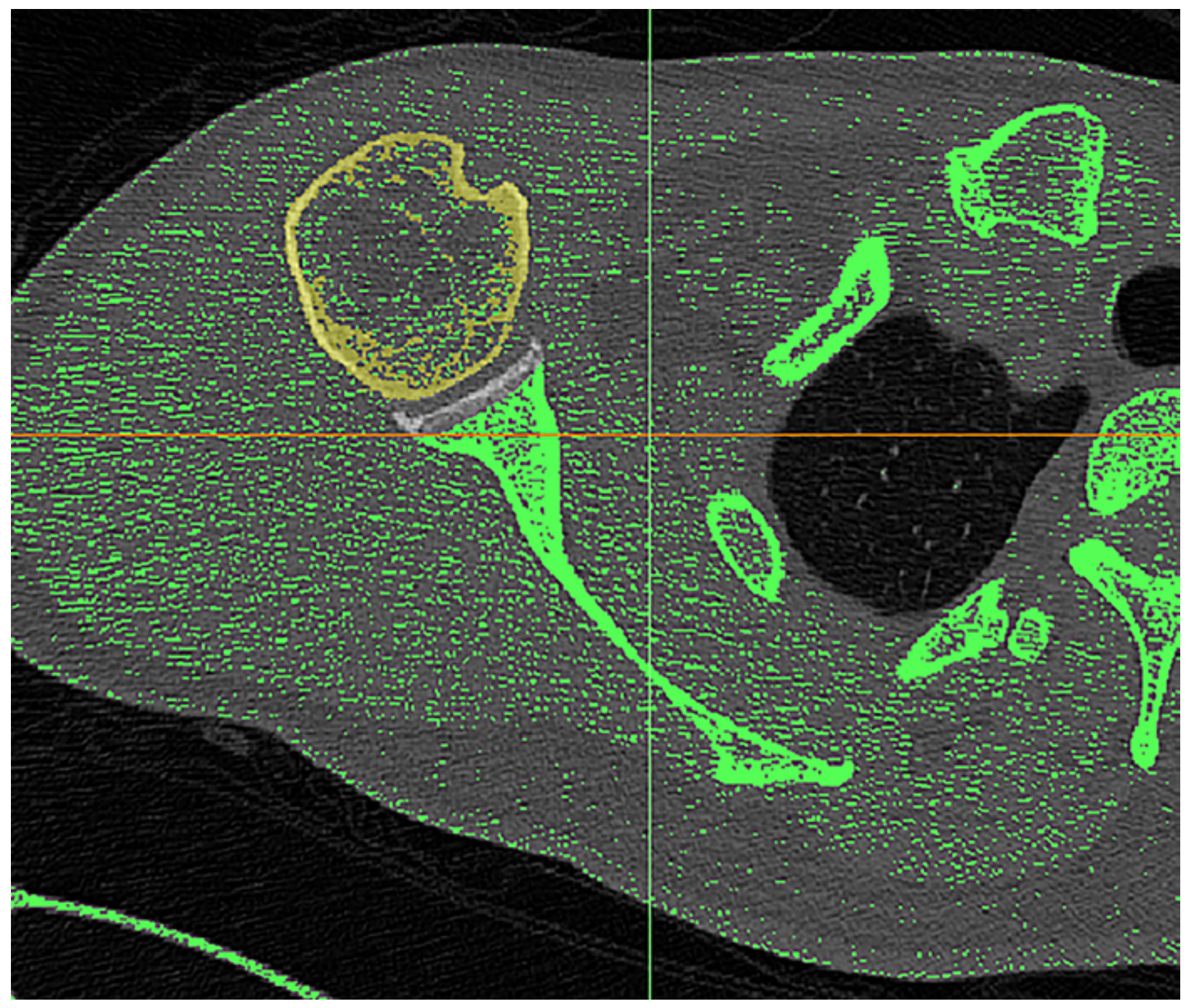

Figure 1

mimics extraction of the humerus 


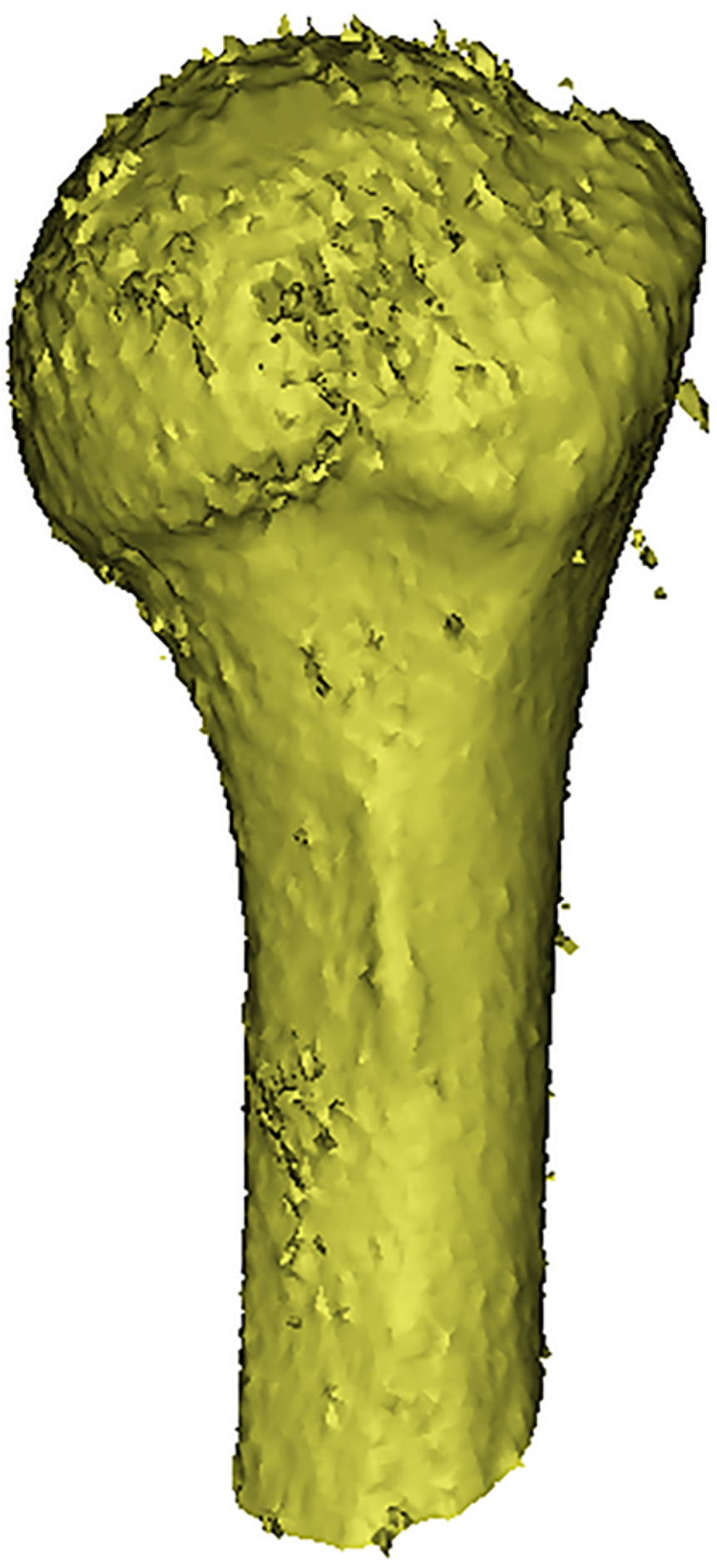

Figure 2

mimics extraction of the humerus 


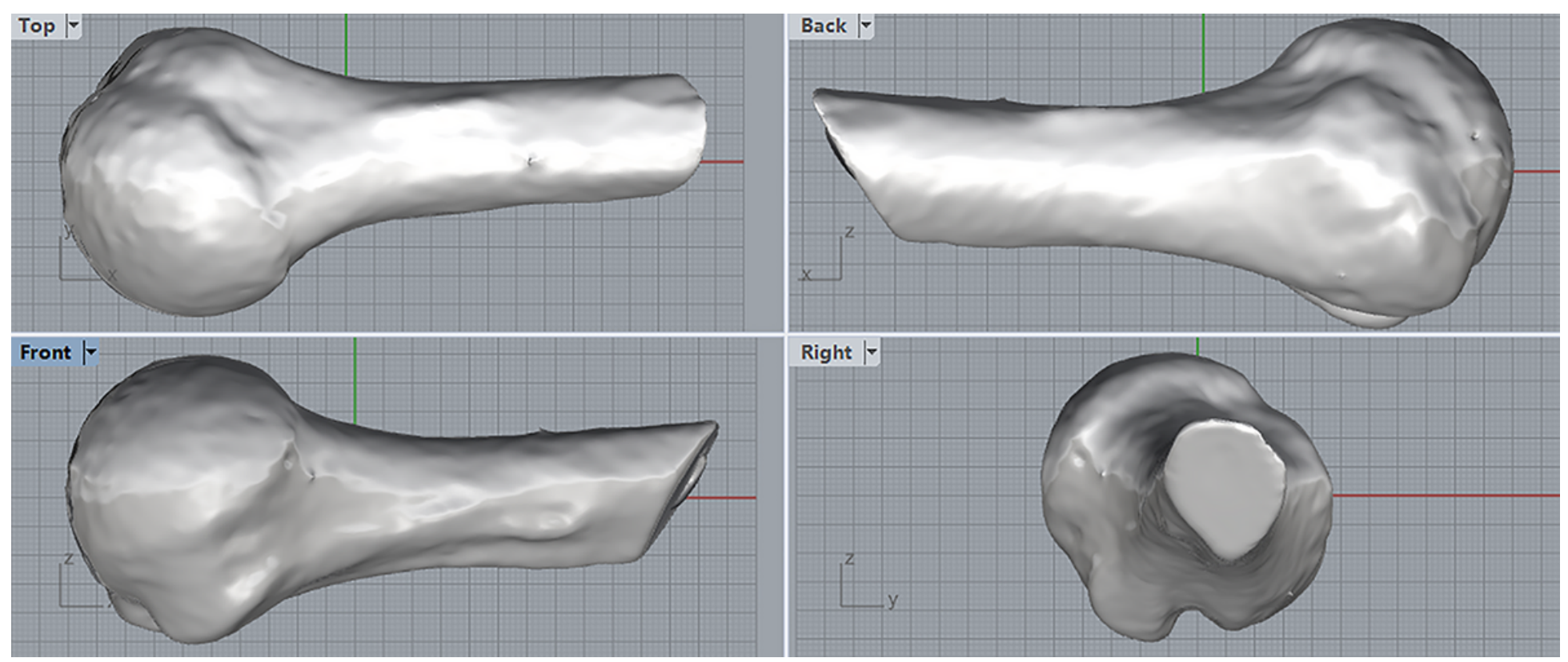

Figure 3

Positioning of the humerus in Rhino

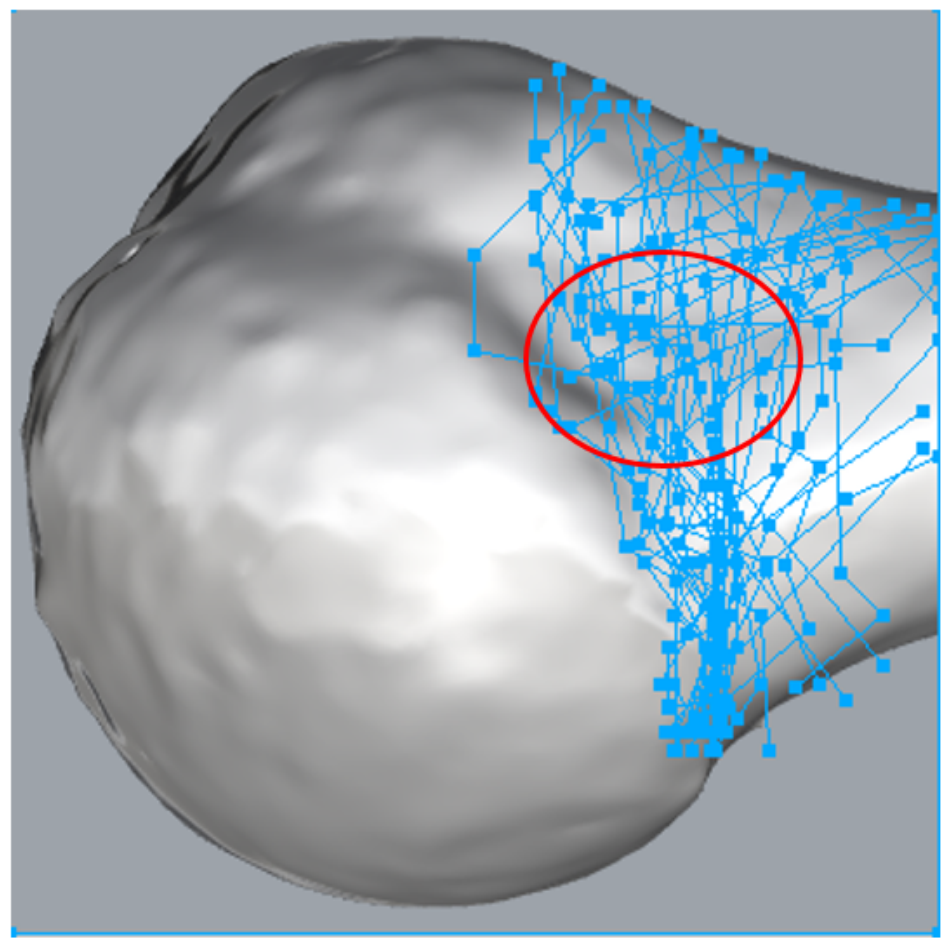

\section{Legend: The areas circled in red are areas of concentration of turning points}


Figure 4

Fracture mapping冈the areas circled in red are areas of concentration of turning points

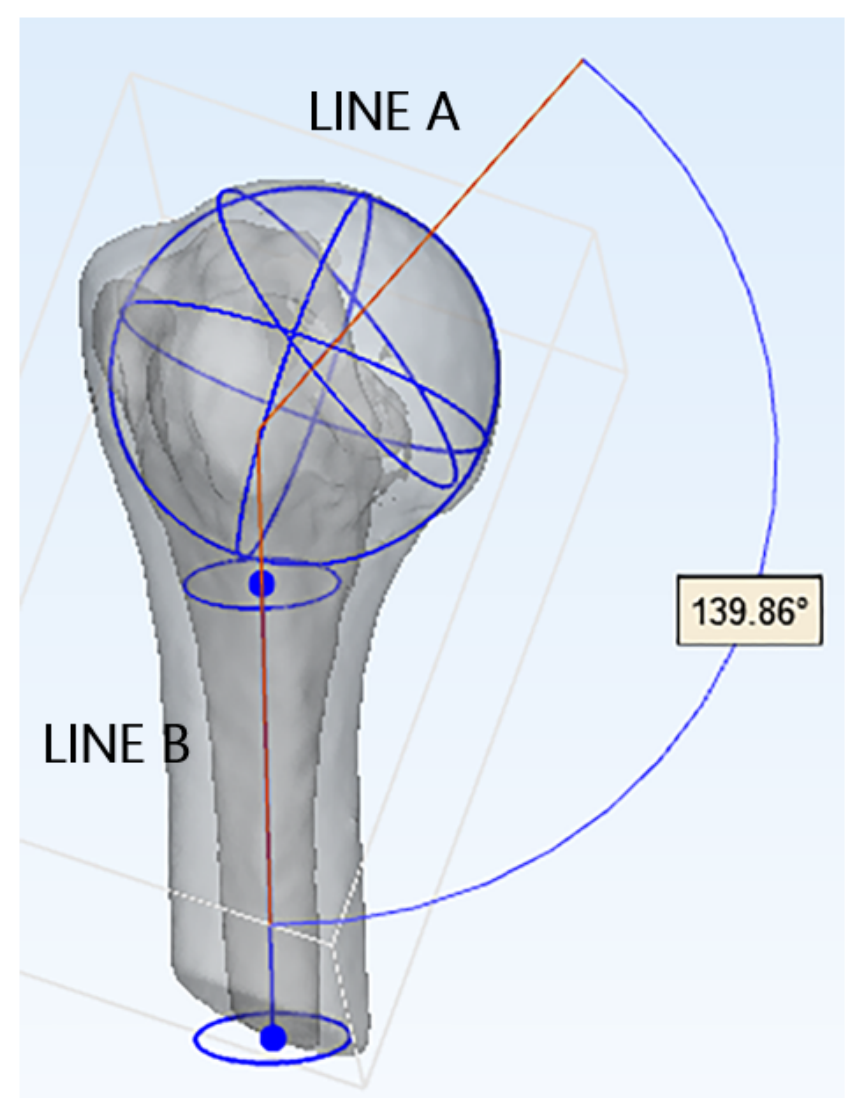

\section{Legend:Spatial angle measurement}

Figure 5

Spatial angle measurement 


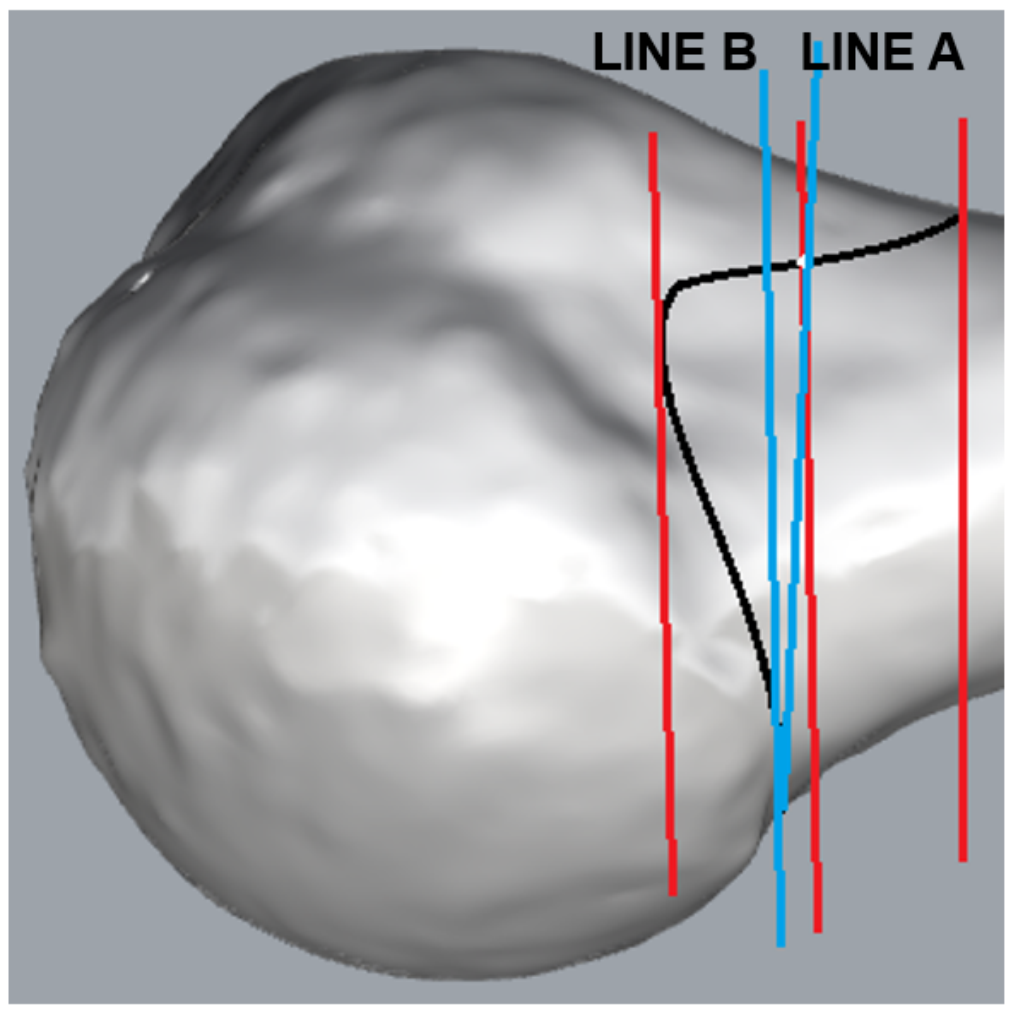

Legend:The black line represents the fracture line and the angle formed by the blue line segment is the angle between the fracture line and the horizontal plane of the humerus

Figure 6

Angle between the fracture line and the horizontal plane of the humerus 


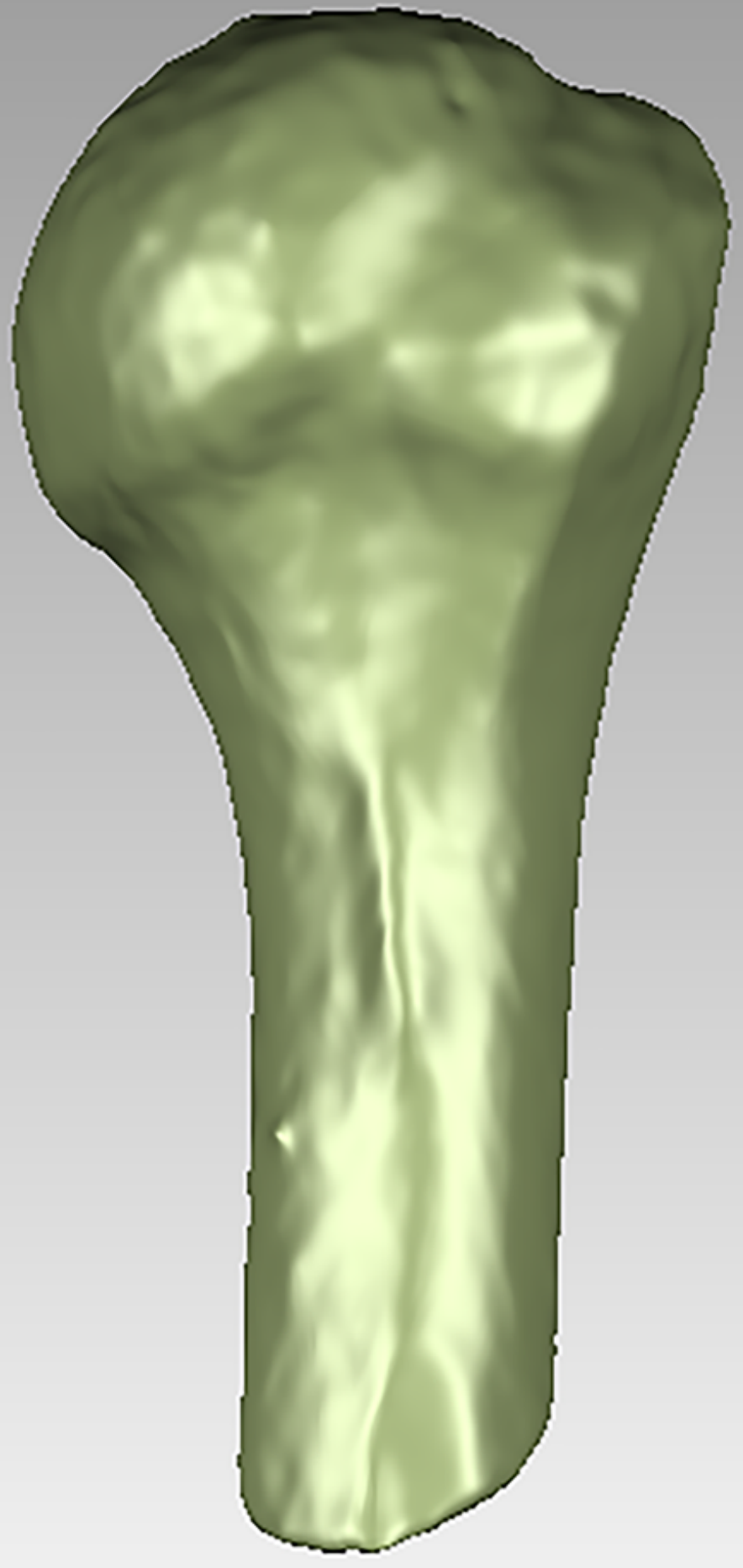

Figure 7

Geomagic retouching 


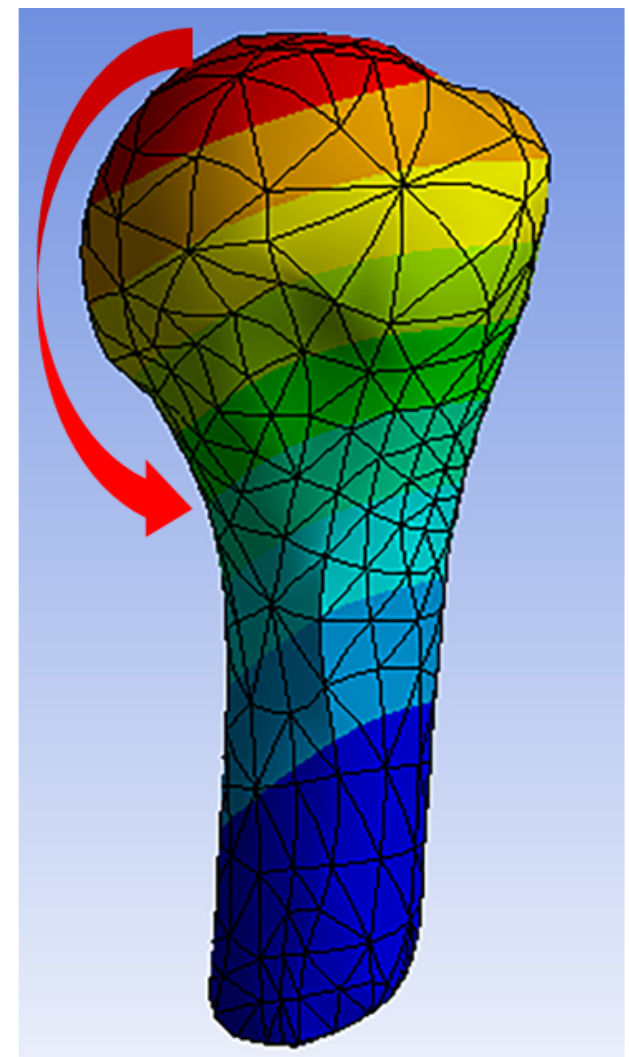

\section{Deformation analysis, the red arrow shows the direction of movement of the humeral head as the force increases}

Figure 8

Deformation analysis, the red arrow shows the direction of movement of the humeral head as the force increases 


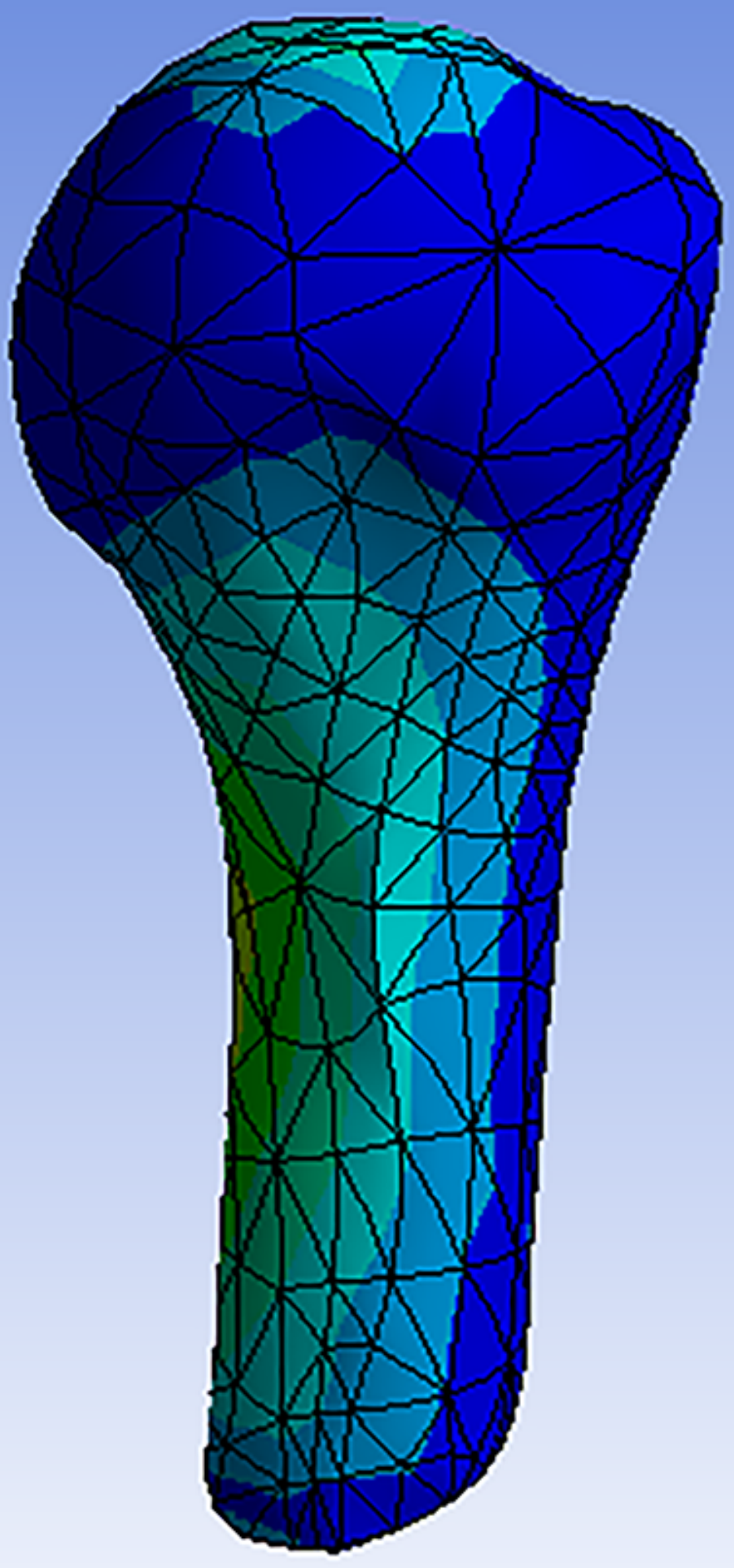

Figure 9

Stress analysis, the darker the blue, the higher the stress 


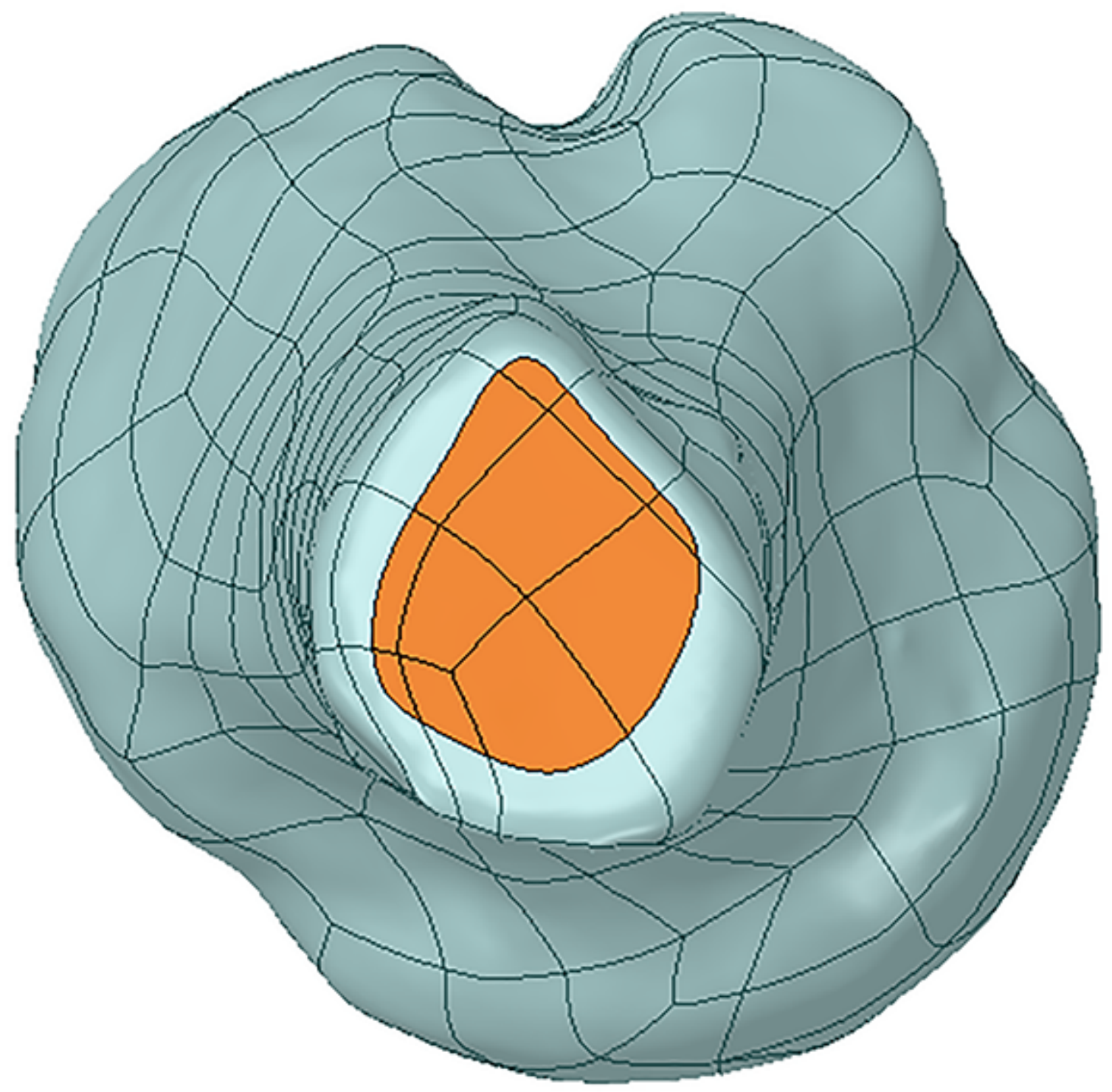

Figure 10

Fixation of the distal humerus 


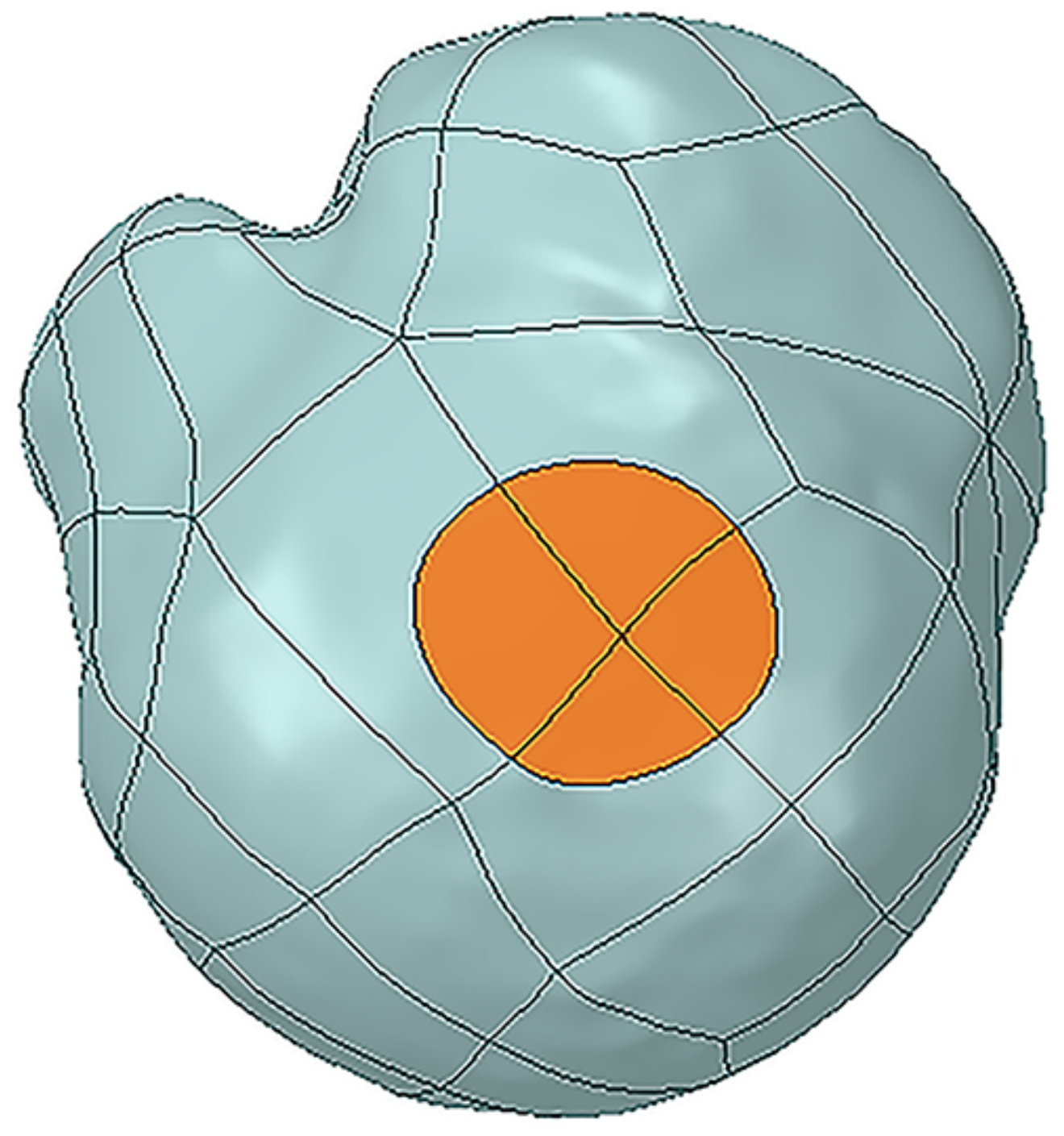

Figure 11

Shoulder joint abduction $90^{\circ}$ force surface 


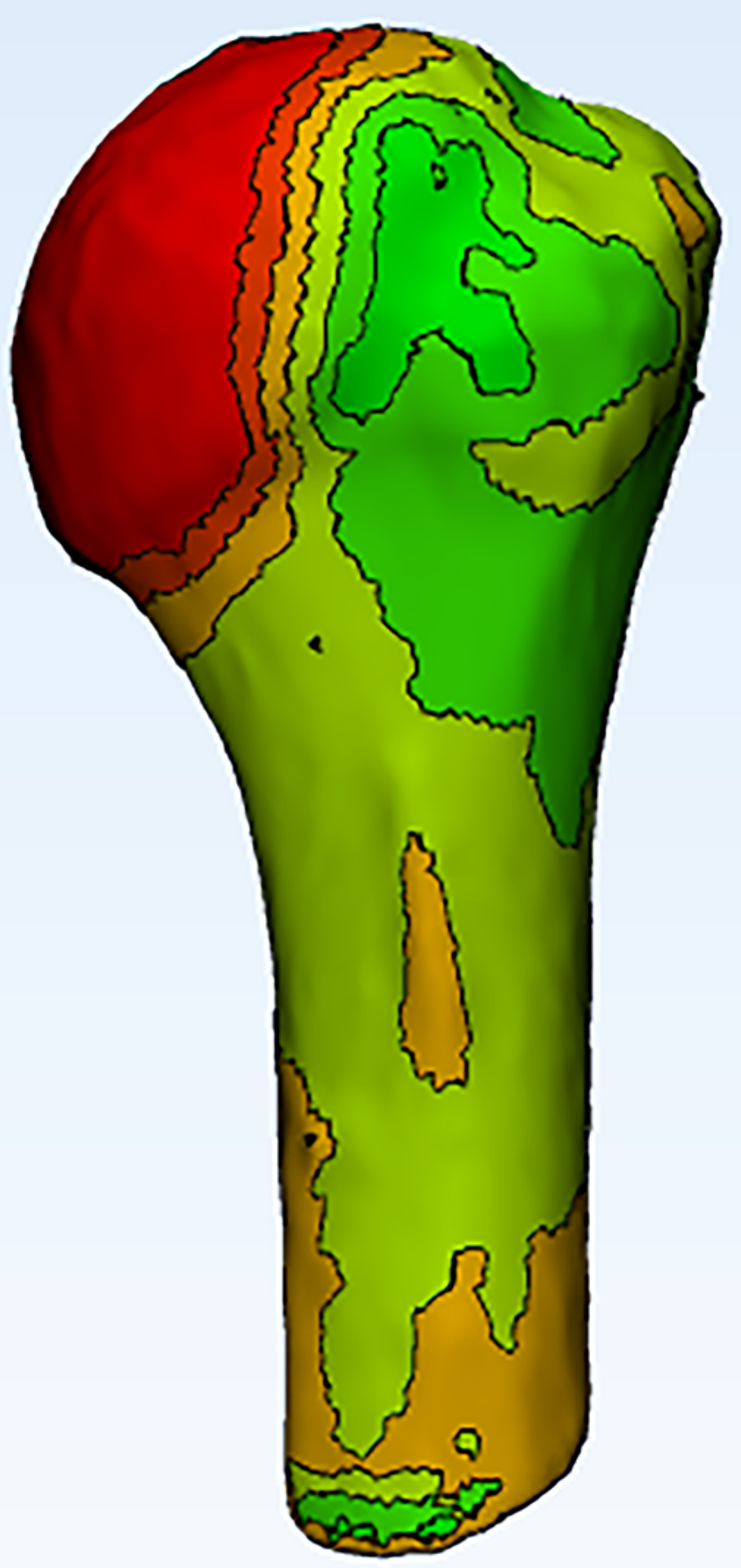

Figure 12

Wall thickness analysis 

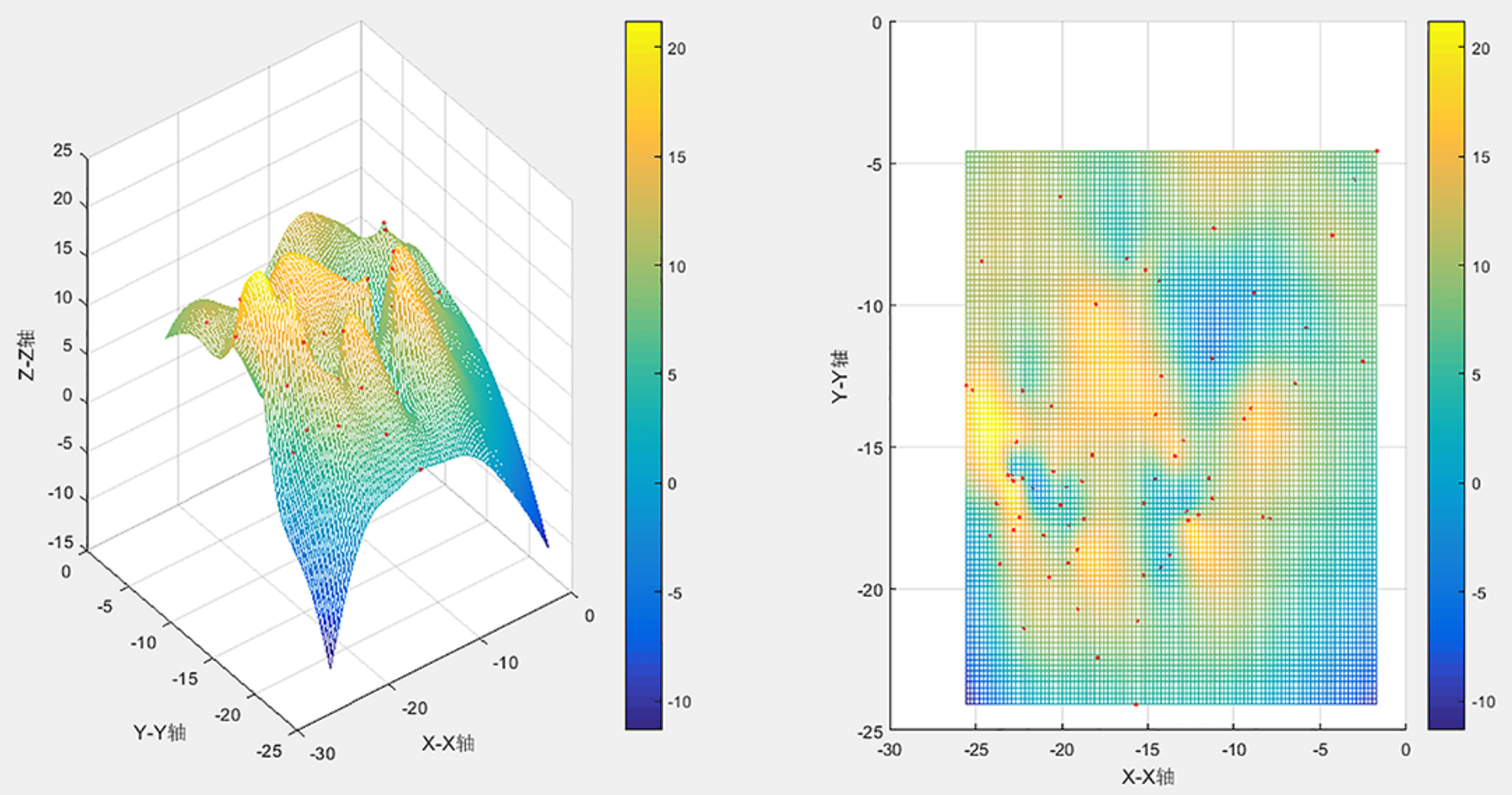

Figure 13

Start and end coordinates matlab heat map analysis, Figure 13 shows the starting point, Figure 14 shows the end point
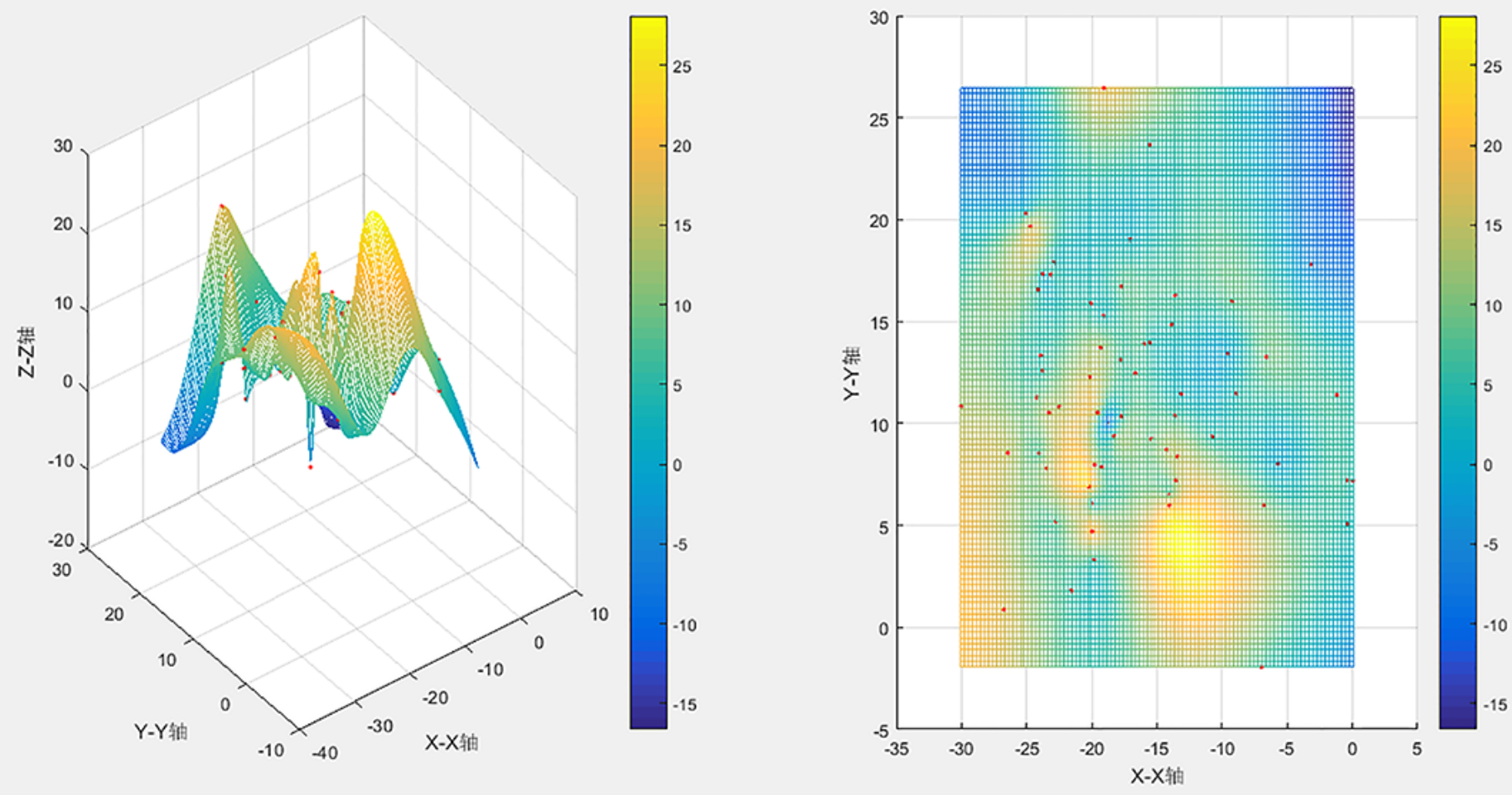

Figure 14 
Start and end coordinates matlab heat map analysis, Figure 13 shows the starting point, Figure 14 shows the end point

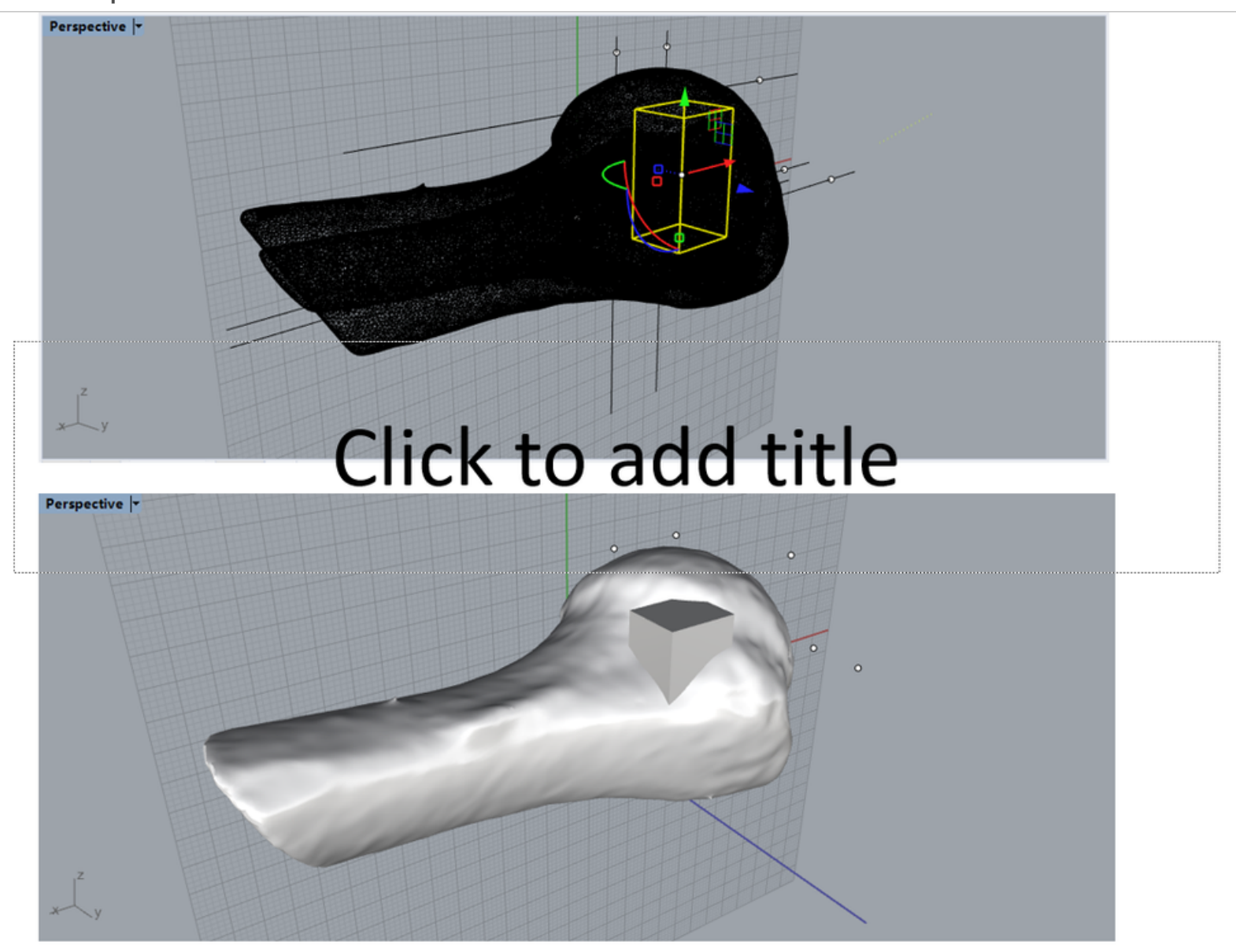

\section{Legend:The coordinates are substituted into the template in Rhino, and the concentrations are circled in red}

Figure 15

Coordinates are substituted into the template in Rhino, with the red circles selected for the substitution of coordinate concentrations 

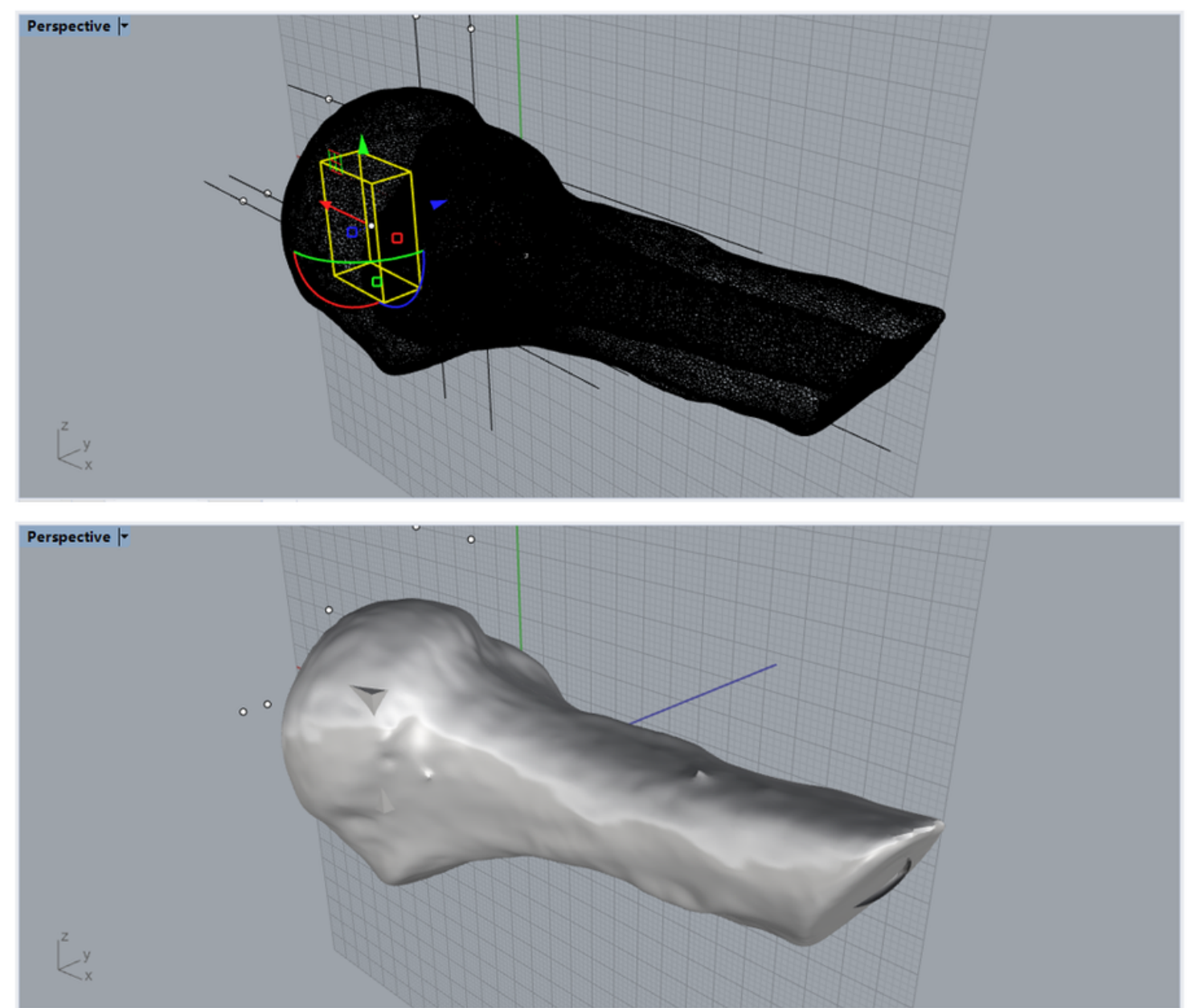

\section{Legend:The coordinates are substituted into the template in Rhino, and the concentrations are circled in red}

Figure 16

Coordinates are substituted into the template in Rhino, with the red circles selected for the substitution of coordinate concentrations 